\title{
Interdisciplinary Interconnections in Synthetic Biology
}

\author{
Ulrich Krohs • Mark A. Bedau
}

Published online: 1 November 2013

(c) Konrad Lorenz Institute for Evolution and Cognition Research 2013

Synthetic biology is not a unified field. It involves many disciplines, from molecular genetics to biochemistry, and from engineering to bioinformatics. It includes xenobiological approaches that build and use artificial nucleic acids, metabolic reengineering of existing microbes, maybe using standardized parts and devices, creation of minimal organisms, bio-brick-biochemistry, and attempts to make synthetic organisms from scratch. The contributions to this thematic issue reflect on these scientific activities in synthetic biology and in related fields. They grew out of a workshop on the scientific, philosophical, and social dimensions of synthetic biology, including its research strategies, conceptual apparatus and societal implications.

For many, the attraction of synthetic biology is its aim to apply in biology the methods of traditional engineering, especially rational engineering, using standardized components that allow plug-and-play modular design (Canton et al. 2008; Mutalik et al. 2013a, b). Rational engineering contrasts with creating desired systems through trial-and-error experimentation, ${ }^{1}$ which includes tried-and-true methods like tinkering and kludging, as well as sophisticated methods like generating and screening massive libraries of high-throughput wet-lab experimental data. Trial-and-error methods in synthetic biology also include familiar and widespread methods like in vitro (or directed) evolution (Yokobayashi et al. 2002), as well as new applications of machine-learning

U. Krohs ( $\square)$

Department of Philosophy and Zentrum für

Wissenschaftstheorie, University of Münster,

Münster, Germany

e-mail: ulrich.krohs@uni-muenster.de

M. A. Bedau

Department of Philosophy, Reed College, Portland, OR, USA

e-mail: mab@reed.edu methods to make high-throughput experiments maximally intelligent and efficient (Caschera et al. 2010, 2011).

Synthetic biology involves a plurality of experimental research programs. It includes top-down reengineering of existing life forms, for example, to mass-produce products such as pharmaceuticals (Martin et al. 2003) or biofuel (Savage et al. 2008). It also includes bottom-up attempts to create new minimal chemical life or "protocells" using nothing but non-living materials (Rasmussen et al. 2004, 2009a). Both top-down and bottom-up synthetic biology employ the two methods we contrasted above: rational engineering and trial-and-error experimentation. These two distinctions (top-down versus bottom-up, and rational engineering versus trial-and-error experimentation) define four quadrants in Table 1. These quadrants map achievements in synthetic biology and illustrate some of the field's scientific plurality. Least familiar and least developed is the lower right quadrant of trial-and-error methods for bottomup synthetic biology, though some would argue that this quadrant also shows the most upside potential (e.g., Bedau 2013, this issue).

\section{Themes from the Workshop}

The workshop, "Synthesis $(\sigma \delta v \theta \varepsilon \sigma ı \varsigma)$ : Interdisciplinary Interconnections in Synthetic Biology," that is the basis for this thematic issue of Biological Theory was held in September 2011 at the $\mathrm{ZiF}$, Center for Interdisciplinary Research, University of Bielefeld, Germany. The format of

\footnotetext{
1 See, however, Morange (2013, this issue), who shows that taking this dichotomy as a strict and stable distinction of different approaches would be a mistake. We stick to these two paradigms as labels for the main focus within the methodological spectrum in which each synthetic approach is situated.
} 
Table 1 Synthetic biology achievements classified by whether the approach is topdown or bottom-up, and whether they use rational engineering or trial-and-error methods

\begin{tabular}{cll}
\hline & Rational engineering & Trial-and-error \\
\hline $\begin{array}{c}\text { Top-down synthetic biology: create new life } \\
\text { forms by modifying existing life forms }\end{array}$ & Martin et al. (2003) & Yokobayashi et al. (2002) \\
& Gibson et al. (2010) & Haseltine and Arnole (2007) \\
& Temme et al. (2012) & Huang et al. (2008) \\
& Mutalik et al. (2013a, b) & \\
Bottom-up synthetic biology: create life & Szostak et al. (2001) & McCaskill (2009) \\
forms using only nonliving materials & Rasmussen et al. (2003) & Caschera et al. (2011) \\
& de Souza et al. (2009) & \\
& Zhu and Szostak (2009) & \\
\hline
\end{tabular}

the workshop was experimental, and it proved quite successful. The workshop involved extensive interaction and discussion. Presentations were organized into groups of three, and after each group the participants divided into small breakout groups with at most eight participants. Breakout groups enabled everyone to participate actively in the discussion and fostered extended exchanges that would be impossible in a plenum. ${ }^{2}$ After one hour each group presented its main results and open questions to the plenum. The group presentations were followed by an open discussion of some especially interesting ideas from each group. While we make no attempt to recount the full richness of the workshop, we can share some especially salient ideas that emerged from the discussion.

Several of the participants believed that many of today's most important and controversial issues involving synthetic biology will be irrelevant in 20 years. Much scientific and philosophical work involving synthetic biology today is tied to our current incomplete understanding of how to make synthetic life forms. We still are identifying the real challenges in synthetic biology. This makes the current development of synthetic biology — and its philosophical reflection-highly dynamical, quickly generating new concepts, questions, and research programs. Nevertheless, today's discussions can at least serve as a Wittgensteinian ladder to be climbed once and then abandoned. In any case, the future course of synthetic biology is unpredictable. We have no choice but to contribute, wait, and observe how it evolves in the future.

Discussions of synthetic biology often invoke Feynman's dictum: what I cannot create, I do not understand.

\footnotetext{
${ }^{2}$ Participants in the workshop and thus contributors to the ideas sketched in the following were: Speakers: Mark Bedau (Reed College, Portland), Steven Benner (The Foundation for Applied Molecular Evolution, Gainesville), Bernadette Bensaude Vincent (Paris), Joachim Boldt (BIOSS Centre for Biological Signalling Studies, Freiburg), Athel Cornish-Bowden (Marseille), Christophe Malaterre (Paris/Montreal), Michel Morange (Centre Cavaillès, Paris), Michael Sismour (Harvard, Cambridge), Kenneth Oye (MIT, Cambridge), Alfred Pühler (Bielefeld), Kepa Ruiz-Mirazo (Donostia-San Sebastián).

Breakout group leaders: Werner Callebaut (Konrad Lorenz Institute, Altenberg), Jane Calvert (Edinburgh), Ulrich Krohs (Bern/ Münster), and Sabina Leonelli (Exeter).
}

The converse is also interesting: what I can make, I do understand. Of course, this converse is false; I do not necessarily understand everything that I can make. But there is an important and closely related open question: how, when, and why does making something help us understand it? This question is not wholly conceptual but partly empirical. Making might be an important tool in some kinds of understanding. It is at least a kind of reality check. If my target is to create something that behaves in a certain way, and I think I know how to do it, then success or failure will teach me whether or not I did understand it. If I never learn how to make the desired target, I will still at least learn the error of my previous ideas. If I succeed in making the desired target, this can confirm my working hypotheses and suggest new experiments. Experimentally manipulating the synthesized mechanism and observing the resulting behavior is often the best way to discover how it works and why it malfunctions. Because synthetic biology can learn valuable lessons when it fails to engineer life, one workshop participant proposed offering a new "Magnificent Failure Award" at iGEM-the annual student International Genetically Engineered Machine competition.

A possibly fundamental, if not magnificent, failure of several programs within synthetic biology might be disregarding the evolutionary aspect of making engineered life. Synthetic biological systems do evolve, whether it is appreciated by synthetic biologists or not. Most often, scientists try to prevent evolution in order to keep the engineering result undistorted. Some workshop participants favored a completely different approach. Since, on the one hand, evolutionary processes do inevitably occur in engineered life anyway, and on the other, evolution is able to bring about and to stabilize emergent properties, evolutionary processes should to the contrary be deployed in synthetic processes. Appropriate selective regimes should be utilized in getting the desired results by means of evolution, rather than trying to protect unstable engineered systems against evolution.

Synthetic biology and systems biology are often regarded as complementary fields in so far as the first is by definition a synthetic approach, while the other is analytic. 
The concept of synthesis certainly demands further analysis (see Malaterre 2013; Benner 2013, both in this issue). However, even on a basic understanding it is doubtful whether such activities can be associated with the disciplines in a straightforward manner. In a way the labels "analytic" and "synthetic" reflect the overall goals of the disciplines rather than the activities performed. They also reflect what can be called cultural traditions within the fields. They stress which of the activities is looked at as being essential to the research project, and which as being just supportive-though the project depends on one type of activity as essentially as the other. One may regard any synthetic activity within biology as engineering, but it seems desirable to pin down the influence of engineering on the new field. The fundamentally important step to engineering in synthetic biology does not at all seem to be the synthetic activity, which belonged anyway to many of the fields that contributed historically to synthetic biology. Crucial, instead, is the involvement of engineers, of scientists with a different kind of training, with different cultural traditions and different thought styles (Fleck [1935] 1979) rather than the adoption of their strategies.

There is a tall tale about the distinction between synthetic biology and systems biology, according to which systems biology is holistic but synthetic biology is reductionist. The truth is different; emergence (of the right form) and reductionism (of the right form) are not contraries, but may each hold with regard to the very same system. This reduction/emergence duality is a central and illuminating feature of both systems and synthetic biology. Many systems biologists follow a hard-core reductionist research agenda while also analyzing holistic effects. Synthetic biology displays an analogous ambivalence. It adds and recombines individual parts in a plug-and-play reductionist manner, but the desired properties it aims to engineer are typically holistic behavior of the global system. This kind of top-down synthetic biology follows a reductionist control paradigm, but the goal is to engineer life forms with desired emergent properties. Synthetic (and systems) biology thus must wed emergence with control, holism with reductionism. It is an open question whether an analogous reduction/emergence dualism is present in all instrument-driven or data-intensive research.

Synthetic biology may help clarify our ideas about what life is. It certainly provides tools to explore and better understand the complexity of life. The epistemic value of succeeding in bottom-up reconstruction of living entities could hardly be overestimated. Nevertheless, as the considerations on the converse of Feynman's dictum show, success at synthesis does not guarantee understanding. Interest in the what-is-life question might dwindle a future biology that swings back and forth between analysis and synthesis, and between epistemic and practical goals. The workshop discussions also questioned how much success at synthetic biology would teach us about the origins of life, and it remained an open question, for example, whether xenobiology might clarify and help answer this question, or whether clarity would come only by investigating evolutionary processes directly.

To judge by the workshop participants, there is widespread agreement about the indispensability of strict risk management in synthetic biology, especially with regard to voluntary and involuntary release into nature of partly or fully synthetic organisms. These worries align with the ethical issues concerning genetic engineering. Workshop participants thought xenobiology projects were less risky than projects using only standard biotic components. Others, however, stressed serious potential risks of a xenobiosphere in the long run. Even on a short timescale, compounds from primary and secondary xeno-metababolism can have unknown and unwanted effects on our environment. Our treatment of possible synthetic organisms themselves also raises some in-principle ethical questions. Can completely artificial organisms inherit their moral status from natural organisms that already exist? Is the concept of a completely artificial organism even coherent? These and related questions reappear throughout the literature on the social and ethical implications of synthetic biology (Bedau and Parke 2009; Kaebnick and Murray 2013).

\section{Contributions to this Issue}

The articles in this issue discuss many overlapping topics, but three main issues stand out. One involves the philosophy of science and engineering, and asks about the distinctive role and value in synthetic biology of the methodologies of rational engineering and trial-and-error experimentation. The second involves epistemology and philosophy of science, and asks about the epistemic and scientific benefits (and costs) of following synthetic biology's distinctive synthetic goals. The third involves metaphysics and ethics, and asks whether synthetic biology implies that simple life forms are merely complex chemical mechanisms that lack any inherent value.

Most authors are well aware that synthetic biology involves both rational engineering and trial-and-error experimentation, including indirect evolutionary methods. Morange (2013, this issue) concludes that the distinction between rational engineering and tinkering is a false contrast, and that continual progress in synthetic biology requires tinkering as much as rational engineering. Giese et al. (2013, this issue) conduct an empirical, bibliometric study to measure to what extent published work in synthetic biology is explicitly characterized as rational engineering rather than tinkering or the like, and 
conclude that there is much more to synthetic biology than rational engineering. Bedau (2013, this issue) argues that trialand-error methods are so central in synthetic biology that they drive the key scientific challenges and opportunities in the field.

A second main issue here arises from the suggestion that the distinctive methodology of synthetic biology is synthesis itself, and asks whether synthesis has any special epistemic advantages for science. The synthetic method of synthetic biology contrasts with the analytic method of much of traditional biology, which understands systems by taking them apart into their components and identifying how each component works. Malaterre (2013, this issue) gives a careful analysis of different kinds of synthesis processes, different kinds of knowledge produced by science, and different kinds of failed synthesis experiments, and concludes that concrete synthesis has the distinctive benefit of enabling us to search at will in huge biochemical possibility spaces that have sparse natural examples. Benner (2013, this issue) goes further and argues that the distinctive synthetic method of synthetic biochemistry has a special scientific power to reveal the causes of natural phenomena, and that failed syntheses are especially valuable for revealing unnoticed fundamental scientific assumptions that turn out to be false. Bedau (2013, this issue) also emphasizes the crucial role of synthesis in discovering and understanding the desired emergent properties of synthetic life forms.

The third main issue concerns a cluster of metaphysical and ethical implications of the synthetic method of synthetic biology. Does synthetic biology imply that simple life forms lack any inherent value, any autonomous aims or purposes, but instead are nothing more than complex chemical machines? Many (cf. Bensaude Vincent 2013, this issue) view synthetic biology as a contemporary manifestation of a mechanistic perspective on life with roots in Descartes. We know that the chemical mechanisms in even the simplest known life forms are extremely complex, involving thousands of reactions among thousands of different chemical species. But when synthetic biologists are creating simple life forms in the laboratory using only non-living raw materials bought from a chemical supply house, it seems obvious that simple life forms are nothing more than a complexly organized and interacting aggregation of molecules; the physical and chemical properties of the molecules seem sufficient to generate and explain all the behavior characteristic of simple life forms. Of course, the molecules in an organism do not exist in a heap; they exist only as part of a complex, self-organizing and self-maintaining web of chemical interactions, of the sort discussed by Ruiz-Mirazo and Moreno (2013, this issue), Bedau (2013, this issue), and Cornish-Bowden (2013, this issue). Bensaude Vincent (2013, this issue) identifies the Cartesian mechanistic view of life embodied by synthetic biology as the root of important ethical concerns about synthetic biology, including its myopic limitation of ethical concerns to assessing risks and finding their technological fixes. Bensaude Vincent prefers a Kantian perspective in which every life form is an end in itself with intrinsic value that does not derive from us but deserves our respect, and she urges an informed public discussion of these broader ethical concerns.

Boldt (2013, this issue) develops Arendt's notion of fabrication and uses it to interpret the synthetic method of synthetic biology. Boldt concludes that fabricators tend to presume that they can and may destroy their creations, because their creations are merely complex chemical machines that were created as means to human ends. They have no inherent value in themselves, because they have no "life of their own." Ruiz-Mirazo and Moreno (2013, this issue) share Boldt's skepticism that simple life forms are merely complex machines, and they emphasize the autonomy exhibited by chemical systems that have a robust ability to maintain themselves because they produce all of the constituent molecules (enzymes, lipids, etc.) that regulate and coordinate their activities. Cornish-Bowden et al. (2013, this issue) discuss a specific mathematical and computational model of a robust, self-maintaining chemical system. Ruiz-Mirazo and Moreno also bet that only open-ended evolution actually explains the complex biochemical organization of autonomous, robustly self-maintaining natural living systems. Autonomous chemical systems depend on certain intricate causal structures with self-repairing cyclic dynamics. The actual evolution of life has benefited from billions of years of evolution that tune and tweak the chemical dynamics that generate robust, self-maintaining chemical systems exhibiting open-ended evolution. It remains an open question whether in only a few years synthetic biology can synthesize new chemical systems with appropriate dynamics that do as well or better.

\section{Conclusion}

Synthetic biology is still in its infancy, and so are our efforts to understand and evaluate its important philosophical implications. The articles in this issue illustrate, investigate, develop, and evaluate many of synthetic biology's interdisciplinary interconnections. They report from the front lines of contemporary discussion. These essays convey many of the main open questions about synthetic biology's research strategies, core concepts, and ethical implications, and they develop promising directions for future investigation.

Reflecting on synthetic biology is not only relevant to the field itself. It also helps clarify more fundamental aspects of biological research. The question of whether synthesis and 
analysis should be regarded as distinct methods, the assessment of the contributions of engineering approaches to huge-data science, and the interrelationship of making and understanding are all urgent to discuss as well with regard to other fields of bioscience. Answers with regard to synthetic biology should be double-checked with problems of other fields and disciplines. The ethical discussion about synthetic biology also has strong connections to and implications for more general ethical problems. For example, the discussion about a possible intrinsic value of synthetic organisms can hardly be ignored by any general discussion of intrinsic value, and any answer given for specific cases within synthetic biology will have implications for the general ethical discourse. So approaching the fundamental and philosophical issues of synthetic biology draws on the one hand on related debates from other fields of research, and it may on the other hand contribute to the methodology and ethics of and within science in general.

Acknowledgments We wish to thank Martin Carrier for funding the workshop that produced this special issue from his DFG Leibniz Award grant and the $\mathrm{ZiF}$ for organizing and hosting it. We wish to thank Werner Callebaut, Jane Calvert, and Sabina Leonelli for leading breakout groups and preparing presentations of the workshop discussions. And we wish to thank Maureen O'Malley, who was centrally involved in the preparation of the workshop but unfortunately was unable to attend.

\section{References}

Bedau MA (2013) Weak emergence drives the science, epistemology, and metaphysics of synthetic biology. Biol Theory 8. doi: 10 . 1007/s13752-013-0139-6

Bedau MA, Parke EC (eds) (2009) The ethics of protocells: moral and social implications of creating life in the laboratory. MIT Press, Cambridge, MA

Benner SA (2013) Synthesis as a route to knowledge. Biol Theory 8. doi: 10.1007/s13752-013-0142-y

Bensaude Vincent B (2013) Ethical perspectives on synthetic biology. Biol Theory 8. doi: 10.1007/s13752-013-0137-8

Boldt J (2013) Life as a technological product: philosophical and ethical aspects of synthetic biology. Biol Theory 8. doi: 10.1007/ s13752-013-0138-7

Canton B, Labno A, Endy D (2008) Refinement and standardization of synthetic biological parts and devices. Nat Biotechnol 26:787-793

Caschera F, Gazzola G, Bedau MA et al (2010) Automated discovery of novel drug formulations using predictive iterated high throughput experimentation. PLoS ONE 5:e8546

Caschera F, Bedau MA, Buchanan A et al (2011) Coping with complexity: machine learning optimization of cell-free protein synthesis. Biotechnol Bioeng 108:2218-2228

Cornish-Bowden A, Piedrafita G, Morán F, et al. (2013) Simulating a model of metabolic closure. Biol Theory 8. doi: 10.1007/s13752013-0132-0

de Souza TP, Stano P, Luisi PL (2009) The minimal size of liposomebased model cells brings about a remarkably enhanced entrapment and protein synthetic. Chem Bio Chem 10:1056-1063
Fleck L (1979) Genesis and development of a scientific fact. Chicago University Press, Chicago. German orig. 1935

Gibson DG, Glass JI, Lartigue C et al (2010) Creation of a bacterial cell controlled by a chemically synthesized genome. Science 329:52-56

Giese B, Koenigstein S, Wigger H, et al. (2013) Rational engineering principles in synthetic biology: a framework for quantitative analysis and an initial assessment. Biol Theory 8. doi: 10.1007/ s13752-013-0130-2

Haseltine EL, Arnole FH (2007) Synthetic gene circuits: design with directed evolution. Annu Rev Biophys Biomol Struct 35:1-15

Huang J, Koide A, Makabe K, Koide S (2008) Design of protein function leaps by directed domain interface evolution. Proc Natl Acad Sci USA 105:6578-6583

Kaebnick GE, Murray TH (2013) Synthetic biology and morality: artificial life and the bounds of nature. MIT Press, Cambridge, MA

Malaterre C (2013) Synthetic biology and synthetic knowledge. Biol Theory 8. doi 10.1007/s13752-013-0136-9

Martin VJJ, Pitera DJ, Withers ST et al (2003) Engineering a mevalonate pathway in Escherichia coli for production of terpenoids. Nat Biotechnol 21:796-802

McCaskill J (2009) PACE report: programmable artificial cell evolution. http://www.istpace.org/Web_Final_Report/the_pace_ report/index.html

Morange M (2013) Comparison between the work of synthetic biologists and the action of evolution: engineering versus tinkering. Biol Theory 8. doi: 10.1007/s13752-013-0134-y

Mutalik VK, Guimaraes JC, Cambray G et al (2013a) Quantitative estimation of activity and quality for collections of functional genetic elements. Nat Methods 10:347-353

Mutalik VK, Guimaraes JC, Cambray G et al (2013b) Precise and reliable gene expression via standard transcription and translation initiation elements. Nat Methods 10:354-360

Rasmussen S, Chen L, Nilsson M, Abe S (2003) Bridging nonliving and living matter. Artif Life 9:269-316

Rasmussen S, Chen L, Deamer D et al (2004) Transitions from nonliving to living matter. Science 303:963-965

Rasmussen S, Bedau MA, Chen L et al (eds) (2009a) Protocells: bridging nonliving and living matter. MIT Press, Cambridge, MA

Rasmussen S, Bedau MA, McCaskill JS, Packard NH et al (2009b) A roadmap to protocells. In: Rasmussen S, Bedau MA, Chen L (eds) (2009a) Protocells: bridging nonliving and living matter. MIT Press, Cambridge, MA, pp 71-100

Ruiz-Mirazo K, Moreno A (2013) Synthetic biology: challenging life in order to grasp, use, or extend it. Biological Theory 8. doi: 10. 1007/s13752-013-0129-8

Savage DF, Way J, Silver PA (2008) Defossiling fuel: how synthetic biology can transform biofuel production. ACS Chem Biol 3:13-16

Szostak JW, Bartel DP, Luisi PL (2001) Synthesizing life. Nature 409:387-390

Temme K, Zhao D, Voigt CA (2012) Refactoring the nitrogen fixation gene cluster from Klebsiella oxytoca. Proc Natl Acad Sci USA 109:7085-7090

Yokobayashi Y, Weiss R, Arnold FH (2002) Directed evolution of a genetic circuit. Proc Natl Acad Sci USA 99:16587-16591

Zhu TF, Szostak JW (2009) Coupled growth and division of model protocell membranes. J Am Chem Soc 131:5705-5713 